\title{
Theoretical Study of Midwave Infrared HgCdTe nBn Detectors Operating at Elevated Temperatures
}

\author{
Nima Dehdashti Akhavan, Gregory Jolley, Gilberto A. Umana-Membreno, Jarek \\ Antoszewski and Lorenzo Faraone \\ School of Electrical, Electronic and Computer Engineering, The University of Western \\ Australia, 35 Stirling Highway, Crawley, WA 6009 \\ Email: nima.dehdashti@uwa.edu.au
}

\begin{abstract}
We report a theoretical study of mercury cadmium telluride ( $\mathrm{HgCdTe})$ unipolar n-type/barrier/n-type (nBn) detectors for midwave infrared (MWIR) applications at elevated temperatures. The results obtained indicate that the composition, doping, and thickness of the barrier layer in MWIR HgCdTe nBn detectors can be optimized to yield performance levels comparable with those of ideal $\mathrm{HgCdTe} p-n$ photodiodes. It is also shown that introduction of an additional barrier at the back contact layer of the detector structure $\left(\mathrm{nBnn}^{+}\right)$leads to substantial suppression of the Auger generation-recombination (GR) mechanism; this results in an order-of-magnitude reduction in the dark current level compared with conventional $\mathrm{nBn}$ or $p$ - $n$ junction-based detectors, thus enabling background-limited detector operation above $200 \mathrm{~K}$.
\end{abstract}

Index Terms - Mercury cadmium telluride ( $\mathrm{HgCdTe})$, Unipolar barrier detector, $\mathrm{nBn}$ detector, $\mathrm{nBnn}^{+}$detector, Device simulation, Midwave infrared (MWIR), Auger suppression, HOT detector. 


\section{INTRODUCTION}

HgCdTe-based photodetectors operating in the mid-wavelength infrared (MWIR; 3-5 $\mu \mathrm{m}$ ) have a broad range of military and civilian applications $[1,2]$. However, the low operating temperature needed for thermal noise reduction requires the use of cumbersome and energyconsuming cooling systems. Research interest in high-operating-temperature (HOT) photodetectors is motivated by the need to substantially reduce the size, weight, and power requirements of infrared imaging systems.

From the perspective of processing and materials science, high-performance MWIR detectors and imaging focal-plane arrays (FPA) have, for decades, been dominated by HgCdTe-based $p$ - $n$ photodiodes $[1,2]$. The long-term success of $\mathrm{HgCdTe}$-based photovoltaic detectors is because of the excellent optoelectronic properties of $\mathrm{HgCdTe}$ and relatively mature deviceprocessing technology. However, the performance of these devices is susceptible to Shockley-Read-Hall (SRH) generation-recombination centers in the depletion region, nonuniformities and/or impurities, and surface leakage current. The last of these demands effective surface passivation, which adds to the complexity of the detector-fabrication process. The development of $\mathrm{HgCdTe}$-based detector structures which substantially simplify device fabrication processes, or circumvent the above challenges, is of great importance, assuming such designs result in devices which perform as well as or better than state-of-theart $p-n$ junction photodetectors. Nevertheless, recently proposed unipolar barrier detector structures are particularly attractive, because, in principle, the SRH-related dark current is substantially reduced $[3,4]$.

Currently, among different barrier IR detectors (BIRDs), the leading position is occupied by devices called unipolar barrier infrared detectors (UBIRDs) which incorporate a single type of doping ( $n$ or $p$ ) in their structures. $\mathrm{A}^{\mathrm{III}} \mathrm{B}^{\mathrm{V}}$ family compounds (e.g., GaSb, InAs $\mathrm{As}_{1-\mathrm{z}} \mathrm{Sb}_{\mathrm{z}}$ cap 
layers, InAs ${ }_{1-\mathrm{y}} \mathrm{Sb}_{\mathrm{y}}$ active region, $\mathrm{AlAs}_{1-\mathrm{x}} \mathrm{Sb}_{\mathrm{x}}$ barrier) have become the most important in the design of UBIRDs, mainly because of a nearly zero band offset in the valence band; this group of materials does not, however, have optical and electronic properties comparable with those of $\mathrm{HgCdTe}$ [5-9]. Although $\mathrm{HgCdTe}$-based nBn structures do not naturally have a zero valence band offset, BIRD detector designs have been successfully adapted to the $\mathrm{HgCdTe}$ alloy, resulting in technological advantages over the $\operatorname{HgCdTe} p-n$ photodiode [10]. These advantages arise from: simplification of the fabrication process, relaxed requirements for the passivation process, elimination of the potential problems with $p$-type in-situ doping in molecular beam epitaxial (MBE) growth, removal of dopant implantation, and its subsequent activation [11-15]. It is worth noting that some approaches may lead to reduction or even elimination of the natural valence band offset of $\mathrm{HgCdTe}$-based $\mathrm{nBn}$ structures [16-18]. As a consequence, the performance of the $\mathrm{HgCdTe}$ BIRDs can be substantially improved. However, this subject does not fit within the scope of this study and will be investigated independently $[19,20]$.

In this paper we report a study of the performance of BIRD MWIR HgCdTe detectors with a cut-off wavelength of $\lambda_{\mathrm{c}}=5 \mu \mathrm{m}$ and operating in the temperature range of $\mathrm{T}=50-300 \mathrm{~K}$. The temperature and bias voltage dependences of the dark current and detectivity of the $\mathrm{HgCdTe}-$ based $\mathrm{nBn}, \mathrm{nBnn}^{+}$, $\mathrm{pn}$ and $\mathrm{pnn}^{+}$photodiode detectors were studied, including all important GR mechanisms, for example SRH, Auger, radiative, trap-assisted-tunnelling (TAT), bandto-band-tunneling (BTB), and existing mobility models (temperature dependence, electric field dependence, and doping dependence) $[21,22]$. 


\section{SIMULATION AND DEVICE DESIGN}

Numerical calculations were performed by use of the commercially available software Synopsys Sentaurus device-simulation framework. The one-dimensional (1D) model incorporates electrical and optical material properties of $\mathrm{HgCdTe}$ from previously published models, and SRH, Auger, radiative, BTBT, and TAT GR mechanisms. Temperaturedependent, field-dependent and doping-dependent mobility models were included in the steady-state drift-diffusion model at all locations within the device [21, 22]. Dirichlet boundary conditions were implemented at the ohmic contacts, where charge neutrality and equilibrium conditions were assumed. The simulations assume step doping and compositional profiles; series resistance and surface recombination effects were assumed to be negligible. Specific equations and relationships used for calculation of carrier lifetimes and recombination rates are detailed in the Appendix I and Table I. The model was validated by fitting the calculated current-voltage characteristics of the $p$ - $n$ photodiode device to those of established experimental measurements [23-26] and models were developed on the basis of measured data $[23,27,28]$. Furthermore, the generation-recombination rates, and mobility values obtained from 1D modeling were compared with analytical models and available published data for $\mathrm{HgCdTe}$ with $x=0.30$ [28, 29] .

Schematic representations of the UBIRD and photovoltaic structures used in this study, with their energy band profiles, are shown in Figs. 1 and 2. Similar HgCdTe-based UBIRD designs have previously been reported by Velicu et. al, Itsuno et. al. and Martinuk et. al. [25, 30-33]. In this study we included all important GR and mobility models. Optimized data were obtained for MWIR $\mathrm{nBnn}^{+}$and $\mathrm{pnn}^{+}$detectors and it will be shown that the performance of the $\mathrm{nBnn}^{+}$detector can be similar to that of the best available pnn ${ }^{+}$detector technology. 
The simulated $\mathrm{nBnn}^{+}$detector for MWIR applications consists of an n-type contact layer of thickness of $1 \mu \mathrm{m}, n$-type barrier layer (e-Barrier) of $50 \mathrm{~nm}$, to block the majority carrier (electron) flow, an In-doped $\left(N_{D}=10^{15} \mathrm{~cm}^{-3}\right) n$-type $\mathrm{HgCdTe}$ absorber layer of thickness of 10 $\mu \mathrm{m}$ and composition $x=0.30$, and an $n$-type doped exclusion barrier of thickness of $1 \mu \mathrm{m}$ and $\mathrm{x}=0.40$ which blocks minority carrier (hole) injection from the ground contact at all temperatures.

\begin{tabular}{|c|c|c|c|c|}
\hline & Contact & e-Barrier & Absorber & h-Barrier \\
\hline Donor concentration, $\mathrm{N}_{\mathrm{D}}\left(\mathrm{cm}^{-3}\right)$ & $1 \times 10^{15}$ & $1 \times 10^{15}$ & varies & $5 \times 10^{17}$ \\
\hline Composition, $x$ & 0.30 & 0.60 & varies & $X_{\mathrm{abs}} \rightarrow 0.4$ \\
\hline Geometry, $d(\mu m)$ & 1 & 0.050 & 10 & 1 \\
\hline Overlap matrix, $\mathrm{F}_{1} \mathrm{~F}_{2}$ & \multicolumn{4}{|c|}{0.2} \\
\hline SRH lifetime $\tau_{\mathrm{no}}, \tau_{\mathrm{po}}(\mu s)$ & \multicolumn{4}{|c|}{10,1} \\
\hline Trap energy level, $\mathrm{E}_{\text {Trap }}$ & \multicolumn{4}{|c|}{$0.25 \mathrm{Eg}$} \\
\hline Trap concentration, $\mathrm{N}_{\text {Trap }}\left(\mathrm{cm}^{-3}\right)$ & \multicolumn{4}{|c|}{$5 \times 10^{13}$} \\
\hline $\begin{array}{l}\text { Capture cross-section, } \sigma_{\mathrm{n}}, \sigma_{\mathrm{p}} \\
\left(\mathrm{cm}^{2}\right)\end{array}$ & \multicolumn{4}{|c|}{$10^{-15}, 10^{-16}$} \\
\hline $\begin{array}{l}\text { Target incident power density, } \\
\phi\left(\mathrm{W} / \mathrm{cm}^{2}\right)\end{array}$ & \multicolumn{4}{|c|}{50} \\
\hline $\begin{array}{l}\text { Background temperature } \mathrm{T}_{\mathrm{B}} \\
\text { FOV }\end{array}$ & \multicolumn{4}{|c|}{$300 \mathrm{~K}, 20^{\circ}$} \\
\hline
\end{tabular}


(a)

pn

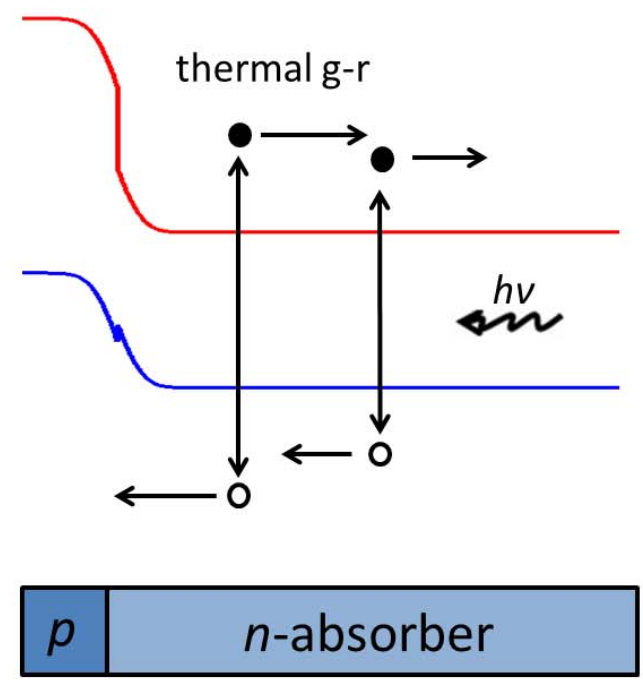

(b)

pnn $n^{+}$

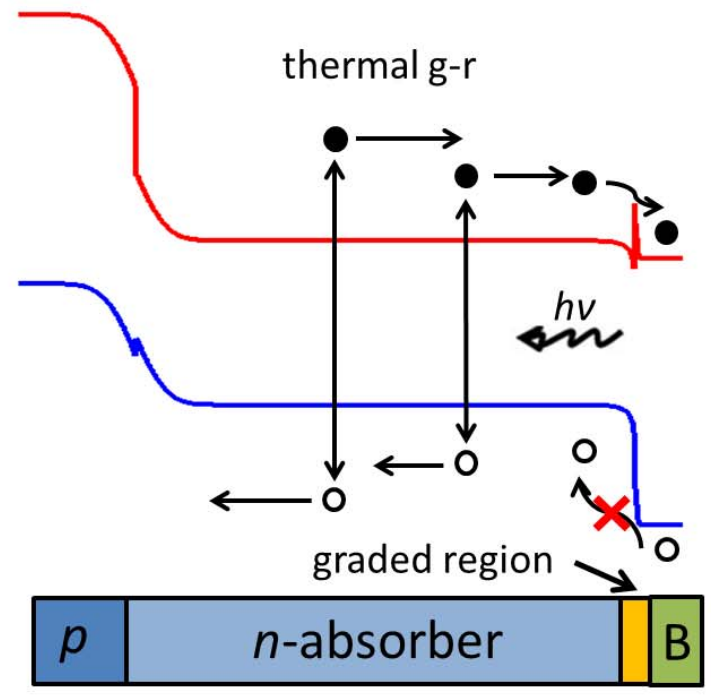

Figure 1. Schematic representation of the $p-n$ photodiode structure and associated energy band diagrams for (a) a $p$ - $n$ photodiode detector, and (b) an HOT $\mathrm{pnn}^{+}$detector with an exclusion junction to block minority carriers.

(a)

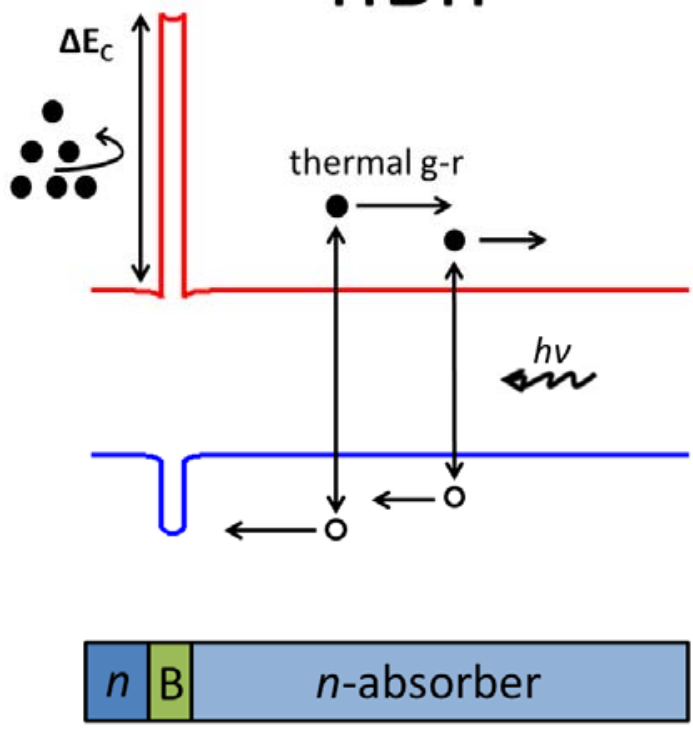

(b)

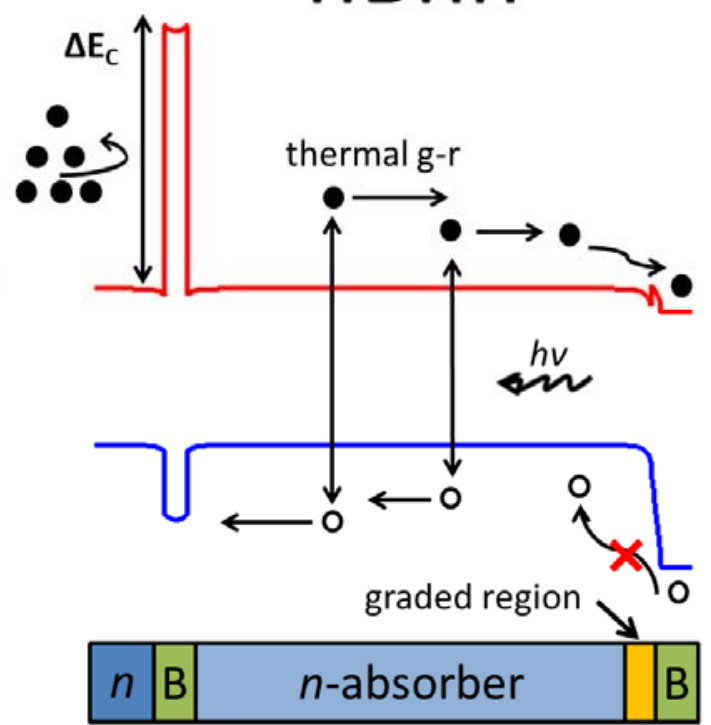

Figure 2. Schematic representation of the UBIRD structure and associated energy band diagrams for (a) an nBn detector, and (b) an $\mathrm{HOT} \mathrm{nBnn}^{+}$detector with an exclusion contact. 


\subsection{Background-Limited Infra-red Performance (BLIP)}

The optical responsivity $(R)$ and detectivity $\left(D^{*}\right)$ were calculated from the following analytical expressions based on current-voltage characteristics. The responsivity $(R)$ was determined by the photocurrent density $J_{\text {photo }}$, because of the incident power density $\varphi$ :

$$
R=\frac{J_{\text {photo }}}{\varphi}
$$

The noise current calculation includes current contributions from thermal Johnson-Nyquist noise and electrical and optical shot noise and is expressed as a quadrature sum:

$$
i_{n}\left(V_{\text {Bias }}\right)=\sqrt{\left(\frac{4 k_{B} T_{d}}{R_{d} A}+2 q\left(J_{\text {Dark }}+J_{\text {Scene }}\right)\right) A}
$$

where $k_{B}$ is Boltzmann's constant, $q$ is electron charge, $T_{d}$ is the detector temperature, $A$ is the detector area, and $R_{d}$ is the dynamic resistance for a given reverse bias. $J_{\text {dark }}$ is the dark current density and $J_{\text {scene }}$ is the wavelength-dependent, scene-induced photocurrent due to the background scene, typically modeled as black-body radiation at $300 \mathrm{~K}$, which can be calculated as follows $[25,32]$ :

$$
J_{\text {Scene }}=2 \pi c q \times \sin ^{2}\left(\frac{\theta}{2}\right) \int_{0}^{\lambda_{s}} \frac{1}{\lambda^{4}} \frac{1}{\exp \left(\frac{h c}{T_{s} k_{B} \lambda}\right)-1} \eta(\lambda) d \lambda
$$

where $\lambda_{c}$ is the cut-off wavelength, $\theta$ is the field of view (FOV) and corresponds to the detector optics, $T_{s}$ is the scene temperature, $\eta$ is the normalized external quantum efficiency, and $h$ and $c$ are Planck's constant and the speed of light, respectively. The electrical noise is typically dominated by thermal noise for operation near zero bias, whereas shot noise will be dominant for the nBn device operated under applied bias. The normalized external quantum efficiency is calculated as a function of the incident radiation wavelength, and can be expressed by: 


$$
\eta(\lambda)=\frac{J_{p h o t o} h c}{\varphi \lambda q}=R \frac{1.24}{\lambda(\mu m)}
$$

where $\lambda$ is the wavelength in $\mu \mathrm{m}$. Finally, the detectivity $\left(D^{*}\right)$ of the device as a function of incident radiation wavelength and current responsivity, is defined as:

$$
D^{*}(\lambda)=\frac{R(\lambda) \sqrt{A}}{i_{n}\left(V_{\text {Bias }}\right)}
$$

where $A$ is the area of the photosensitive region of the detector. The focal ratio $f_{\#}$ of the detector optics is given by $f / D$, where $f$ is the lens focal length and $D$ is the diameter of the entrance pupil. It is related to the FOV, such that a focal ratio $f_{\#}=0$ corresponds to $2 \pi F O V$. Typical $f_{\#}$ values in common infrared detector systems vary between $2\left(\mathrm{FOV} \sim 30^{\circ}\right)$ and 5 (FOV $10^{\circ}$ ) depending on the application. In this study FOV is assumed to be $20^{\circ}\left(f_{\#}=2.835\right)$. The BLIP temperatures for dark current and detectivity of an MWIR detector considered in this study are shown in Figs. 3a and b.
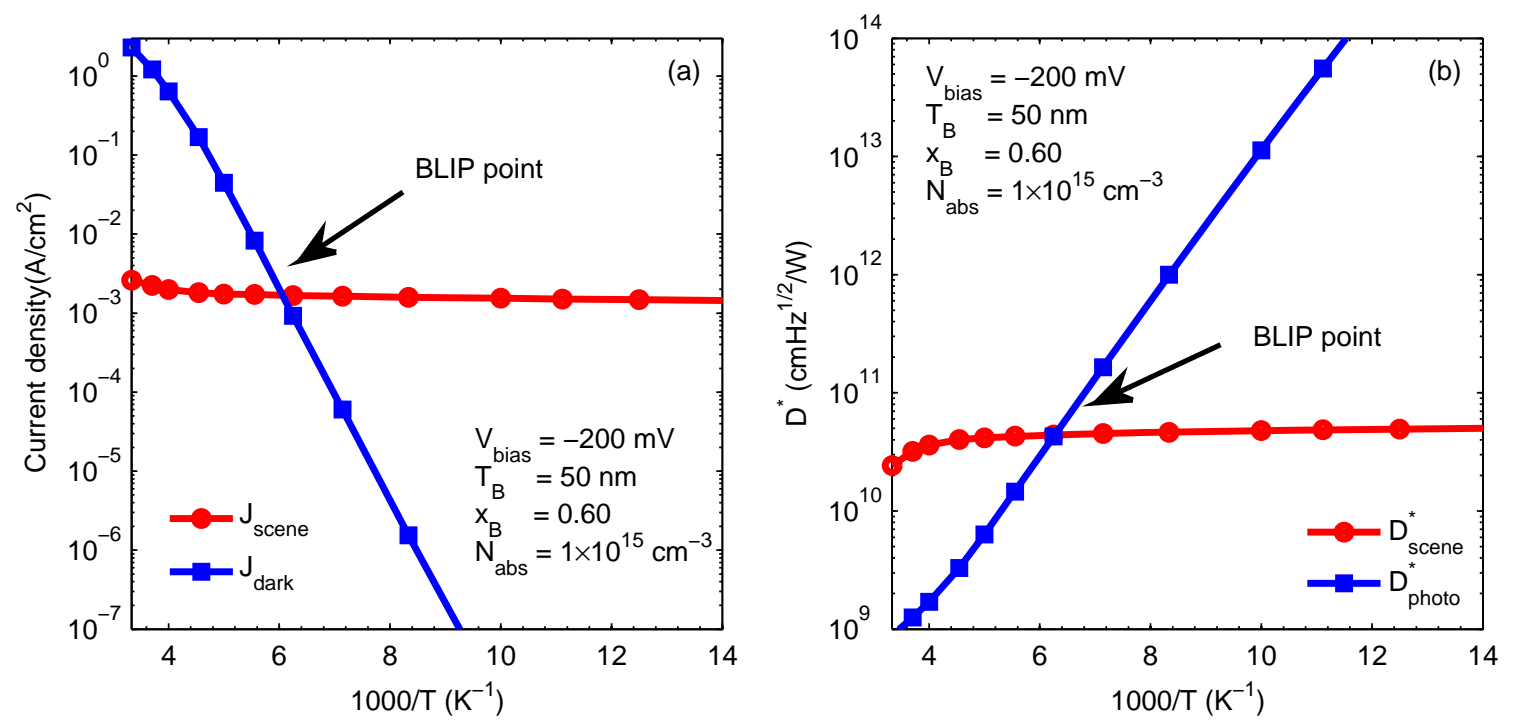

Figure 3. Procedure used to identify the BLIP point in an $\mathrm{nBn}$ detector. (a) The intersection of the scene current and dark current correspond to a BLIP point with a BLIP current density of $2 \times 10^{-3} \mathrm{~A} / \mathrm{cm}^{2}$; (b) the BLIP value of $D^{*}$ is equal to $3.2 \times 10^{10} \mathrm{cmHz}^{1 / 2} / \mathrm{W}$. It is apparent that both the dark current and detectivity reach the BLIP condition at $T=166 \mathrm{~K}$. 


\subsection{Theory of HOT Detectors}

The HOT photon detector concept originally introduced by Elliot and Ashley had a p-i-n ${ }^{+}$ structure in which an intrinsic region contains a $p$-type background dopant [34]. The intrinsic IR absorbing volume is connected to both a minority carrier contact and a majority carrier contact. The proposed device operates in strong non-equilibrium by reverse biasing the minority carrier contact to completely extract all the intrinsically generated minority carriers. Charge neutrality in the absorbing volume is violated, creating an electric field to sweep out the intrinsically generated majority carriers until the majority carrier concentration equals the background dopant concentration. Elimination of the minority carriers throughout the device means that minority carrier recombination processes are eliminated, and the dark current is determined entirely by generation mechanisms within the active volume of the device plus possible components related to injection from contact and surfaces. The HOT detector structures considered in this study were shown in Figs 1 and 2, and the data were specified in Table I.

\section{Optimization of the $\mathrm{nBnn}^{+}$Detector}

\section{1. e-Barrier Optimization}

The barrier composition of UBIRD structures is important in the ultimate performance of the $\mathrm{nBnn}^{+}$detectors. A barrier with a high proportion of Cd leads to a very large valence band offset $\left(\Delta E_{\mathrm{V}}\right)$, which blocks the flow of minority carriers (holes) toward the contact and results in reduced detectivity. In contrast, a low proportion of $\mathrm{Cd}$ in the barrier leads to a very small conduction band offset $\left(\Delta E_{\mathrm{C}}\right)$, compromising its blocking function and, consequently, reduced detectivity. Thus, adjusting the amount of $\mathrm{Cd}$ in the barrier layer is crucial to achieving a high-performance $\mathrm{nBnn}{ }^{+}$detector. 
Figure 4 shows the valence band diagram of an $\mathrm{nBnn}^{+}$detector for different values of the barrier width at $\mathrm{V}_{\text {bias }}=-100 \mathrm{mV}$ and $T=100 \mathrm{~K}$. The optimum barrier width is $50 \mathrm{~nm}$; a barrier layer with wider thickness will require a larger bias to eliminate the valence band offset $\left(\Delta E_{\mathrm{V}}\right)$. On the other hand, a thinner barrier layer will increase quantum mechanical tunneling of the majority carriers (electrons) through the conduction band energy offset $\left(\Delta E_{\mathrm{C}}\right)$ and, therefore; increase the dark current.

Figure 5 shows the energy band offsets in the barrier $\left(\Delta E_{\mathrm{C}}, \Delta E_{\mathrm{V}}\right)$ as function of barrier doping density at a fixed voltage of $\mathrm{V}_{\text {bias }}=-1 \mathrm{mV}$ and $T=100 \mathrm{~K}$, where it is apparent that increasing the barrier doping density results in larger $\Delta E_{\mathrm{V}}$ and smaller $\Delta E_{\mathrm{C}}$ which corresponds to larger dark current. The variation of $\left(\Delta E_{\mathrm{C}}, \Delta E_{\mathrm{V}}\right)$ as a function of barrier doping density is because when the barrier doping density is increased the Fermi level in the barrier becomes closer to the conduction band and, to keep the Fermi energy of the barrier aligned with the Fermi energy in both the absorber layer and contact layer, the top of the conduction band and bottom of the valence band should shift to lower energies.

Figure 6a shows $D^{*}$ as a function of barrier Cd composition and for $T=100 \mathrm{~K}$ and $T=200 \mathrm{~K}$. It is apparent that the $\mathrm{Cd}$ composition should be set to $x=0.60$ to achieve the highest detectivity at both low and high temperatures. Figure $6 \mathrm{~b}$ shows $D^{*}$ as a function of barrier composition and for different bias values. For a maximum flow of minority carriers and, therefore, maximum $D^{*}$, a minimum bias of $\mathrm{V}=-300 \mathrm{mV}$ should be applied to the detector.

Figures $7 \mathrm{a}$ and b show $J_{\text {dark }}$ and $J_{\text {photo }}$ as a funvtion of bias and for different values of Cd composition, $x_{\mathrm{B}}$, at $T=100 \mathrm{~K}$ and $T=200 \mathrm{~K}$. It is apparent that high $x_{\mathrm{B}}$ values reduce $J_{\text {dark }}$ by increasing the valence band offset whereas small values of $x_{\mathrm{B}}$ result in a small conduction band offset and hence large $J_{\text {dark }}$. 


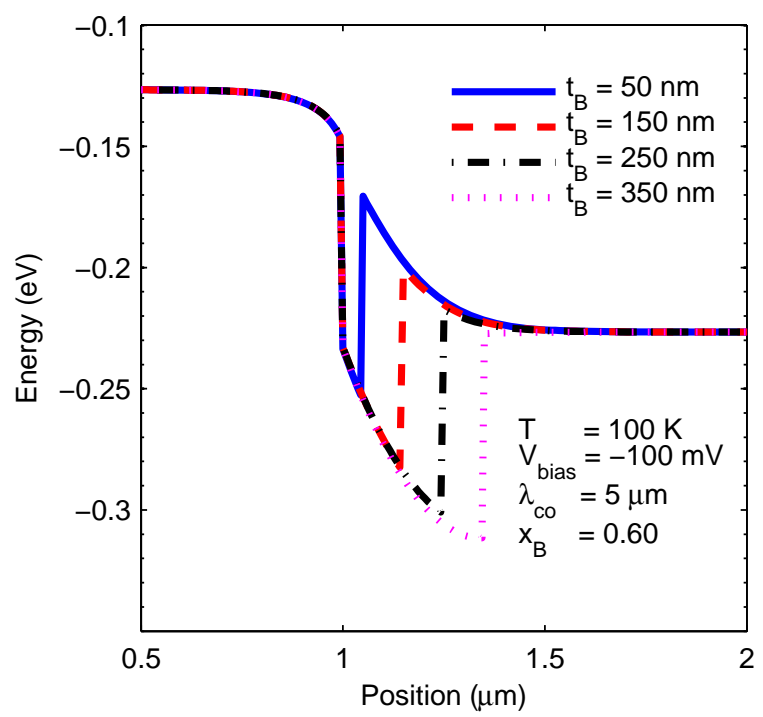

Figure 4. Valence band diagram in for different values of barrier width at $V_{\text {bias }}=-100 \mathrm{mV}$ and $T=100 \mathrm{~K}$.

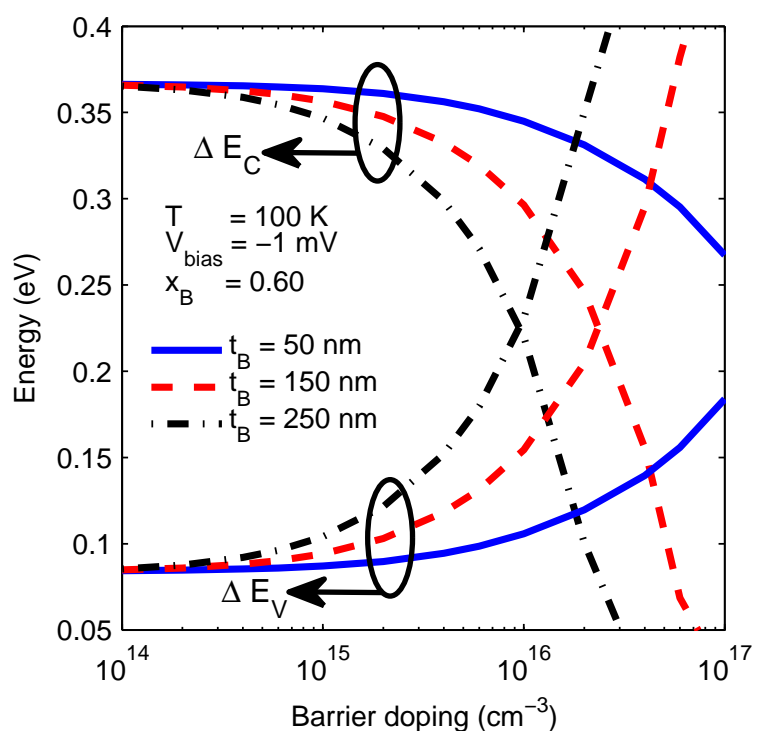

Figure 5. Variation of $\Delta E_{\mathrm{C}}$ and $\Delta E_{\mathrm{V}}$ as a function of barrier doping density at a fixed applied voltage of $\mathrm{V}_{\text {bias }}=-1 \mathrm{mV}$. Increasing the barrier doping density will modify the barrier energy bands in such a way that large $\Delta E_{\mathrm{V}}$ and small $\Delta E_{\mathrm{C}}$ will be observed at very high doping levels. 


\subsection{Absorber Optimization}

Having optimized the barrier composition for the MWIR $\mathrm{nBnn}^{+}$detector, we investigated the effect of absorber layer doping density. In our calculations we assumed the barrier and contact layer doping density at $\mathrm{N}_{\mathrm{abs}}=10^{15} \mathrm{~cm}^{-3}$ and $\mathrm{V}_{\text {bias }}=-400 \mathrm{mV}$ (this $\mathrm{V}_{\text {bias }}$ is in the range where $\mathrm{BTBT}$ is negligible and the same time $\Delta E_{\mathrm{V}}$ is minimum). Figure $8 \mathrm{a}$ shows $D^{*}$ as a function of barrier doping density in the presence of a tunneling mechanism at $T=100 \mathrm{~K}$ and $T=200 \mathrm{~K}$. It is apparent that the absorber doping density should be kept below $2 \times 10^{15} \mathrm{~cm}^{-3}$ to keep $D^{*}$ above the BLIP limit at $T=200 \mathrm{~K}$. When $T=100 \mathrm{~K}$ the detectivity is well above the BLIP limit. Figure $8 \mathrm{~b}$ shows $D^{*}$ as a function of absorber doping density for different values of bias at $T=200 \mathrm{~K}$. A minimum bias of $-200 \mathrm{mV}$ should be applied to the detector to reduce $\Delta E \mathrm{~V}$ and enable the flow of minority carriers.

Figure 9a shows $J_{\text {dark }}$ and $J_{\text {photo }}$ as a function of absorber doping at $T=200 \mathrm{~K}$; it is apparent that the BTBT component starts to increase significantly for doping densities higher than $2 \times 10^{15} \mathrm{~cm}^{-3}$. Figure $9 \mathrm{~b}$ shows $J_{\text {dark }}$ and $J_{\text {photo }}$ as a function of bias for different absorber doping levels at $T=200 \mathrm{~K}$; a reverse bias $>200 \mathrm{mV}$ is required to reduce the valence band offset and enable the flow of minority carriers, whereas absorber doping densities higher than $2 \times 10^{15} \mathrm{~cm}^{-3}$ result in strong BTBT. 

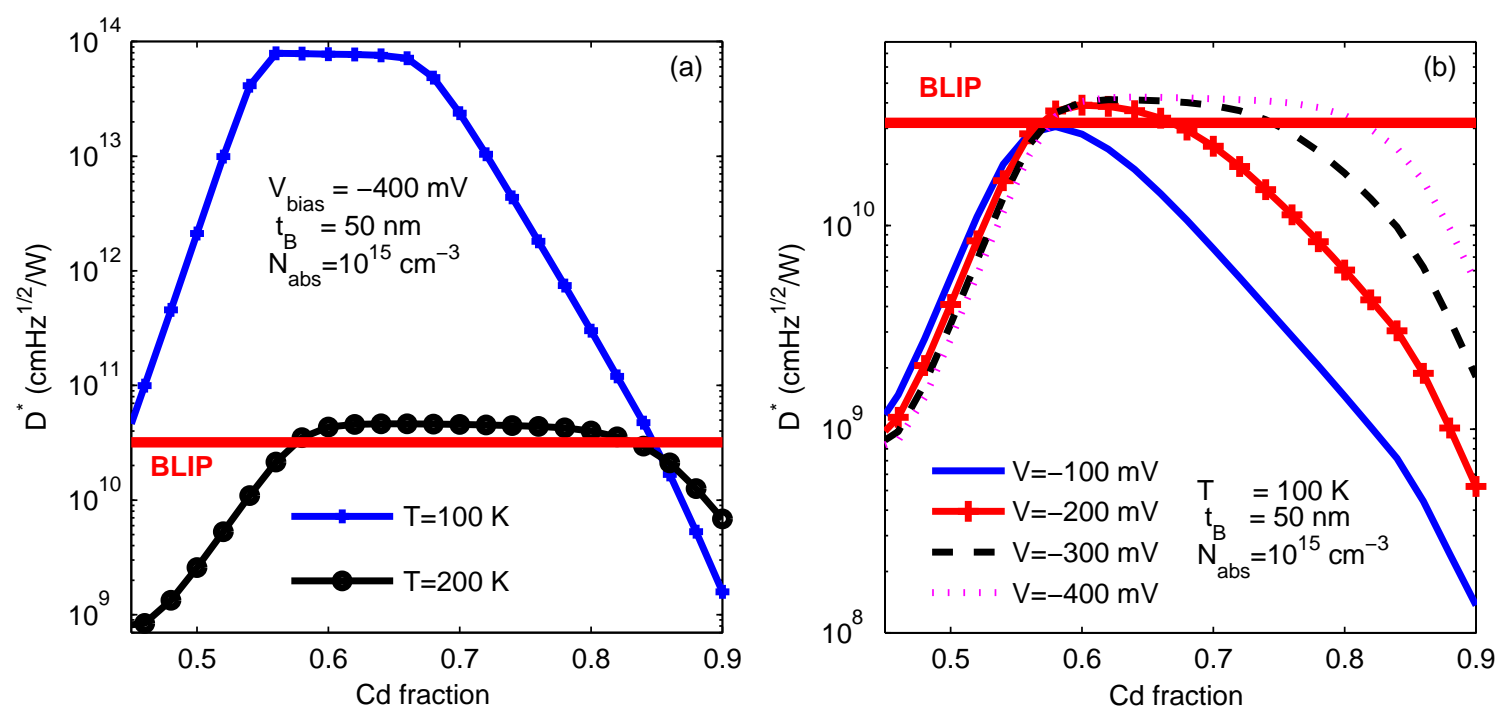

Figure 6. (a) $D^{*}$ as a function of barrier Cd composition for $T=100 \mathrm{~K}$ and $T=200 \mathrm{~K}$ for the $\mathrm{nBnn}^{+}$detector. (b) $D^{*}$ as a function of e-barrier $\mathrm{Cd}$ composition for different values of applied bias for $T=200 \mathrm{~K}$ in the $\mathrm{nBnn}^{+}$detector.
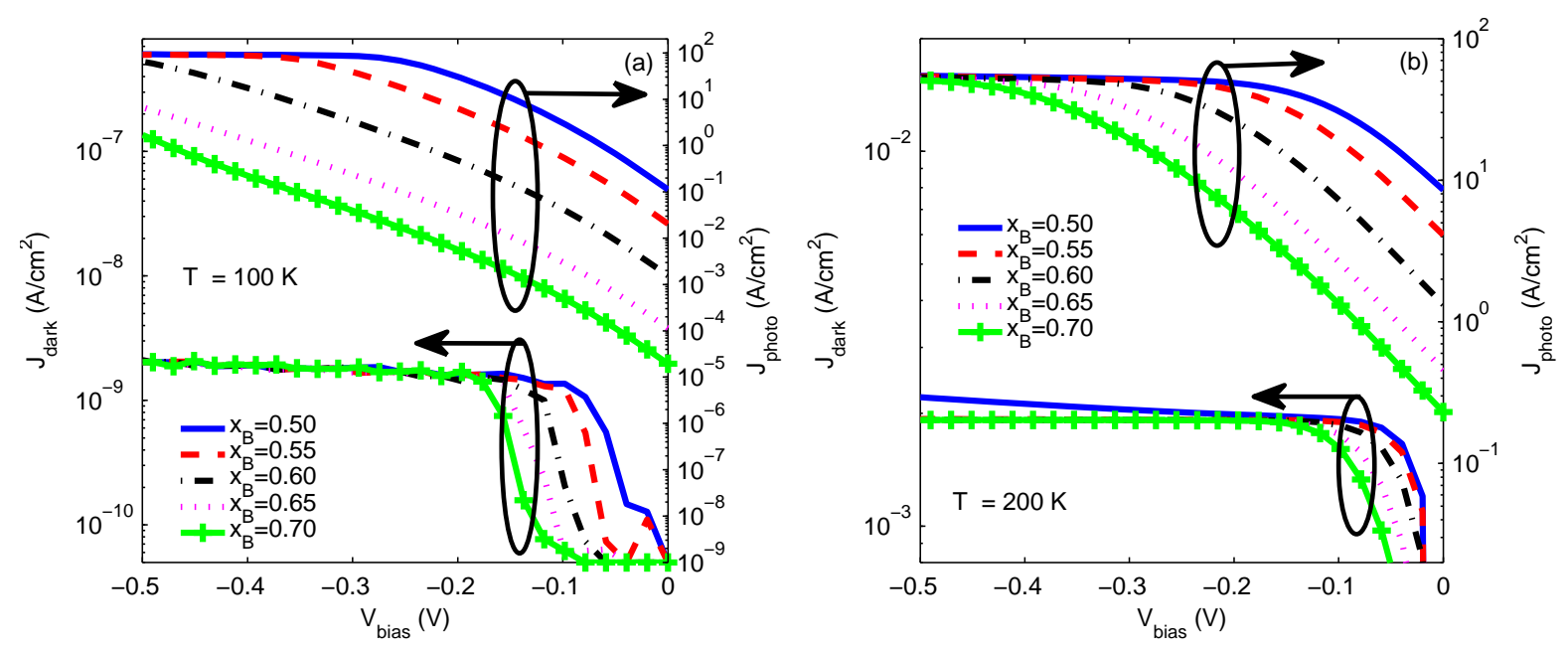

Figure 7. $J_{\text {dark }}$ and $J_{\text {photo }}$ as a function of applied bias for different Cd composition in the barrier for (a) $T=100 \mathrm{~K}$ and (b) $T=200 \mathrm{~K}$. 

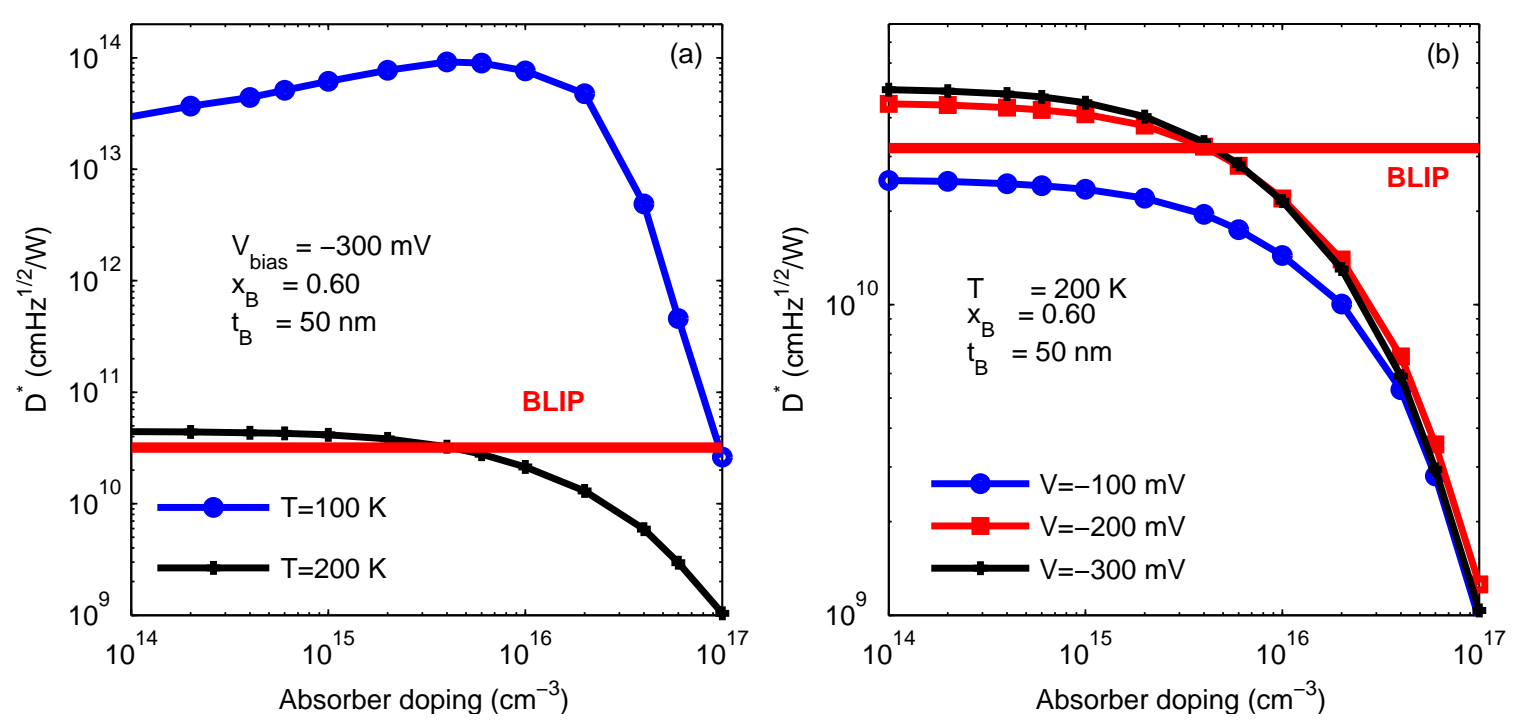

Figure 8. (a) $D^{*}$ as a function of absorber doping density for $T=200 \mathrm{~K}$ and $T=100 \mathrm{~K}$; (b) $D^{*}$ as a function of absorber doping density for different values of bias at $T=200 \mathrm{~K}$.
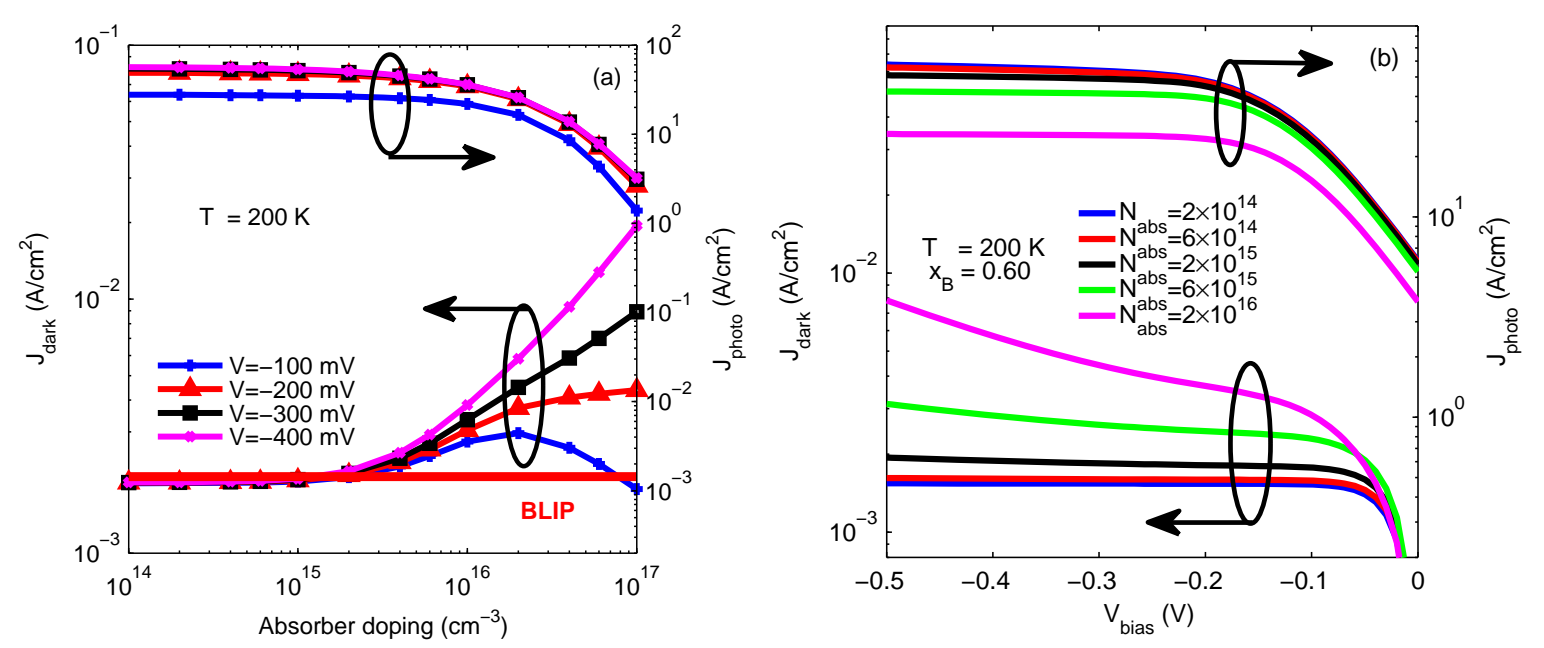

Figure 9. (a) $J_{\text {dark }}$ and $J_{\text {photo }}$ as a function of absorber doping density at $T=200 \mathrm{~K}$ and $\mathrm{x}_{\mathrm{B}}=0.6$ for different values of bias; (b) $J_{\text {dark }}$ and $J_{\text {photo }}$ as a function of applied bias for different absorber doping densities. 


\section{Optimization of the pnn $^{+}$HOT Detector}

\subsection{Optimization of the Depletion Layer}

Unlike the $\mathrm{nBnn}^{+}$detector, for which performance is determined by the properties of the barrier, $\mathrm{pnn}^{+}$detector performance is determined by the pn junction depletion region. Hence, optimization of the contact layer and absorber layer doping densities in $p$ - $n$ photodiode were the main objectives in this part of the work. Figures $10 \mathrm{a}$ and b show $D^{*}$ and $J_{\text {dark }}$ at $T=100 \mathrm{~K}$ and $T=200 \mathrm{~K}$ as a function contact layer doping density ( $p$-type), assuming a fixed absorber doping density of $\mathrm{N}_{\mathrm{abs}}=10^{15} \mathrm{~cm}^{-3}$. It is apparent that the contact layer doping density should be larger than $5 \times 10^{16} \mathrm{~cm}^{-3}$ if $D^{*}$ is to be higher than the BLIB limit. Setting the contact layer doping density above this minimum value creates a depletion region wide enough to collect the photogenerated minority carriers (holes) in the absorber region. Figures $11 \mathrm{a}$ and $\mathrm{b}$ show $D^{*}$ and $J_{\text {dark }}$ as a function of absorber doping density in the presence of different tunneling mechanisms at $T=100 \mathrm{~K}$ and $T=200 \mathrm{~K}$. For $T=200 \mathrm{~K}$, it is apparent that, without considering BTBT, the Auger-1 recombination mechanism will reduce $D^{*}$ for absorber layer doping densities higher than $2 \times 10^{15} \mathrm{~cm}^{-3}$. Addition of BTBT reduces $D^{*}$ even further.

Figures $12 \mathrm{a}$ and $\mathrm{b}$ show $D^{*}$ and $J_{\text {dark }}$ as a function of temperature and for different values of contact layer doping density assuming a fixed absorber doping density of $10^{15} \mathrm{~cm}^{-3}$ for the $\mathrm{pnn}^{+}$detector. As already discussed, reducing the contact doping density will reduce the detectivity of $\mathrm{pnn}^{+}$detectors by reducing the depletion layer width. 

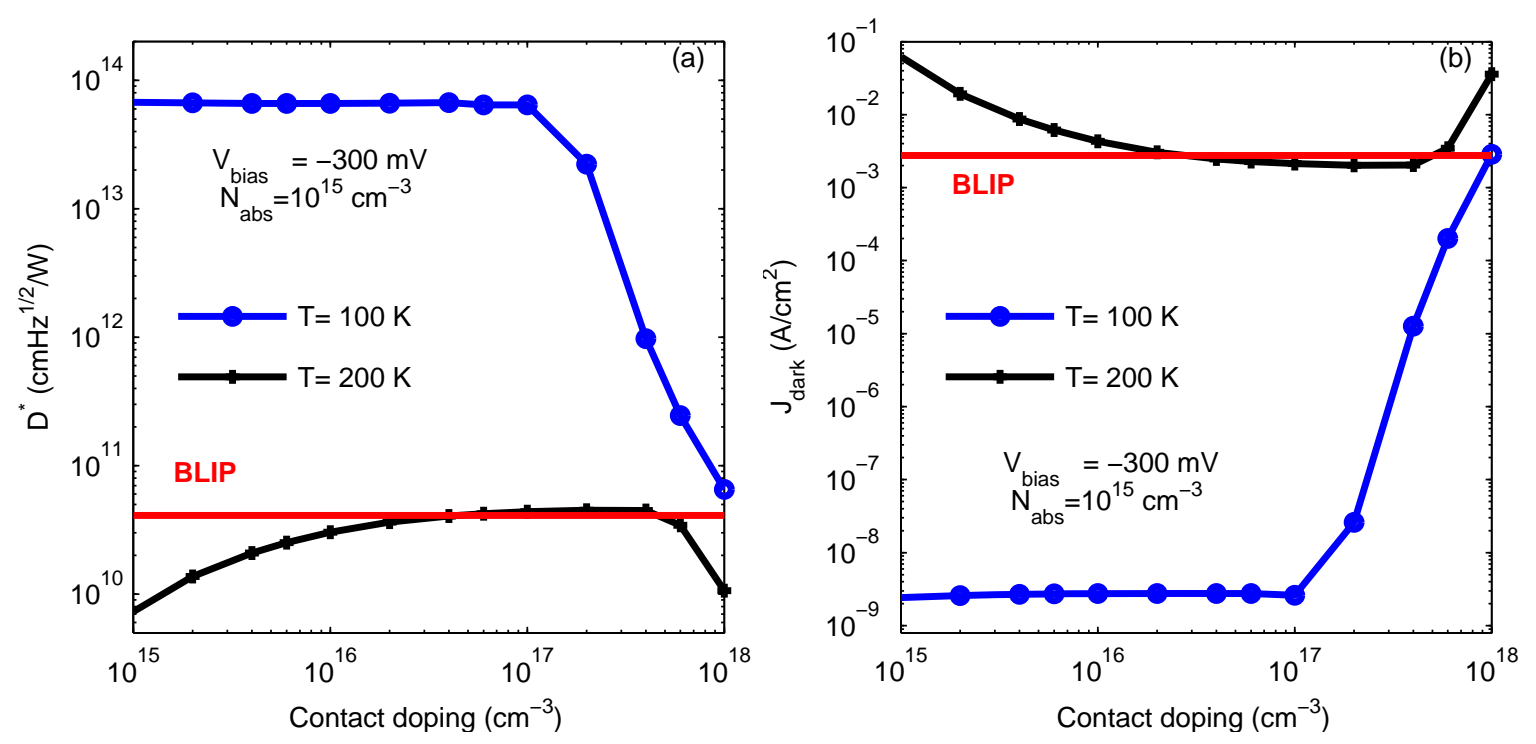

Figure 10. (a) $D^{*}$ and, (b) $J_{\text {dark }}$ as a function of contact layer doping density for $T=100 \mathrm{~K}$ and $200 \mathrm{~K}$ for the $\mathrm{pnn}^{+}$detector. It is evident that BTBT limits the maximum doping density in the contact layer.
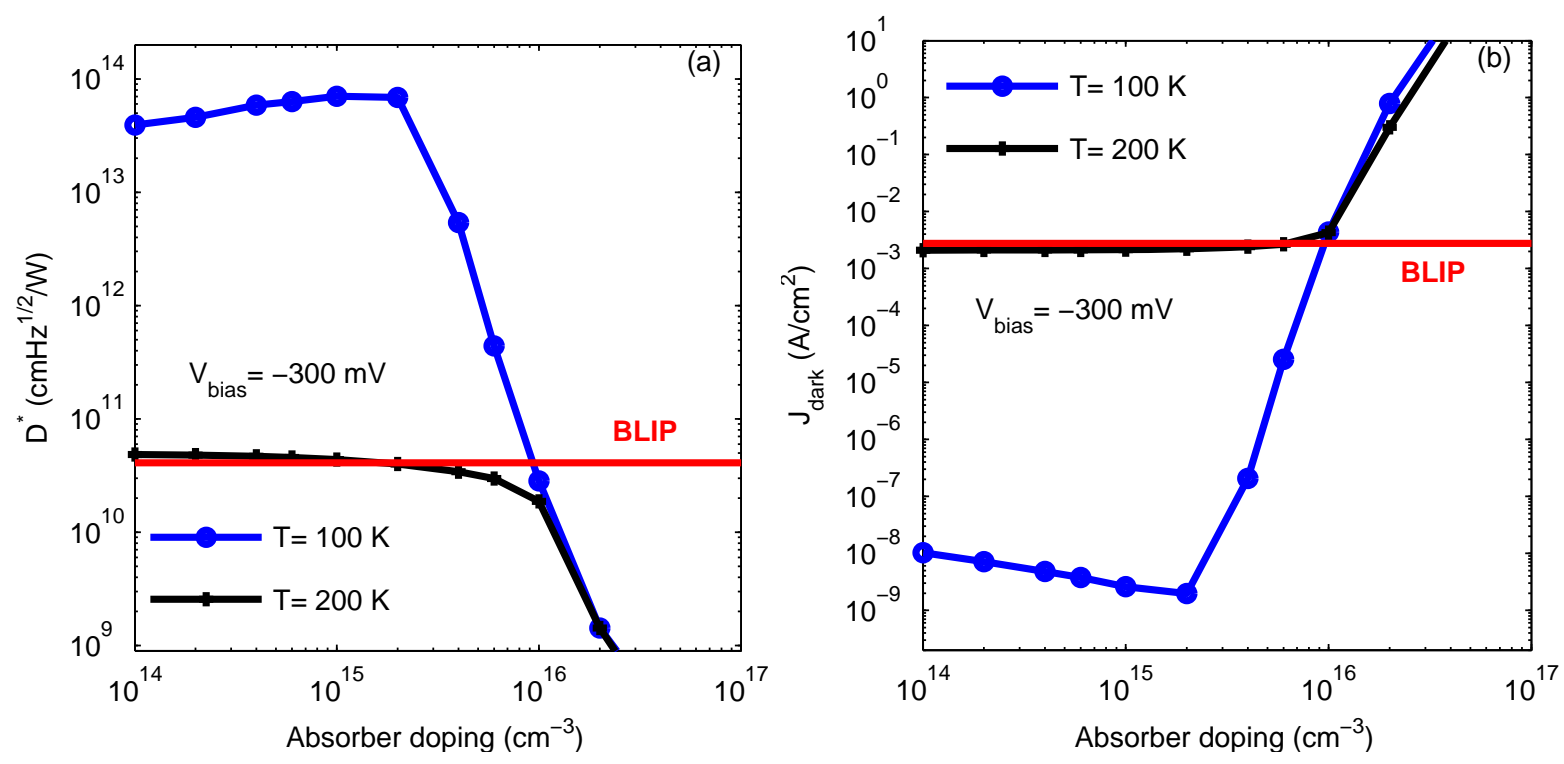

Figure 11. (a) $D^{*}$ and, (b) $J_{d a r k}$ as a function of absorber layer doping density for $T=100 \mathrm{~K}$ and $200 \mathrm{~K}$ for the $\mathrm{pnn}^{+}$detector. It is evident that maximum doping density in the absorber layer is limited by the Auger-1 mechanism. 

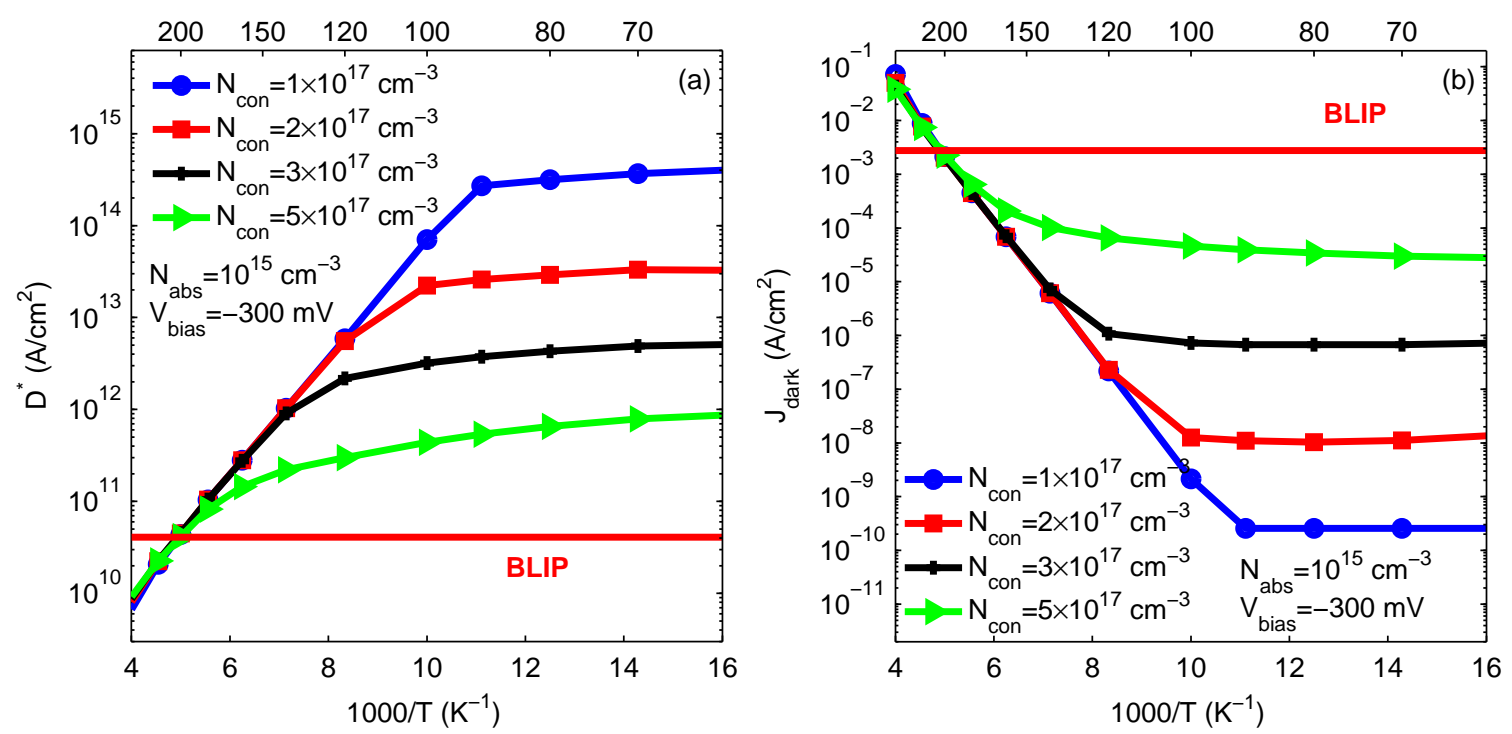

Figure 12. (a) $D^{*}$ and, (b) $J_{d a r k}$ as a function of inverse temperature and for different values of contact layer doping density for the $\mathrm{pnn}^{+}$detector. Absorber doping density is fixed at $\mathrm{N}_{\mathrm{abs}}=1 \times 10^{15} \mathrm{~cm}^{-3}$.

\section{Performance Comparison of HOT Detectors}

In the previous sections we optimized the barrier conditions for the $\mathrm{nBnn}^{+}$and contact/absorber layer conditions for the of $\mathrm{pnn}^{+}$detectors. In this section we compare the performance of $\mathrm{nBn}, \mathrm{nBnn}^{+}, \mathrm{pn}$, and $\mathrm{pnn}^{+}$detectors to determine whether HgCdTe-based barrier detectors have any advantages over $p$ - $n$ photodiode technology. Figures $13 \mathrm{a}$ and 13 show $D^{*}$ and $J_{\text {dark }}$ as a function of absorber layer doping density at $T=100 \mathrm{~K}$ and a fixed reverse bias of $300 \mathrm{mV}$. It is apparent that $\mathrm{nBnn}^{+}$and $\mathrm{pnn}^{+}$photodiodes have the same $D^{*}$ value for low absorber doping density, however, the onset of performance degradation in $\mathrm{pnn}^{+}$because of Auger-1 and BTBT starts earlier compared with nBnn ${ }^{+}$.

Figures $14 \mathrm{a}$ and 14 show $D^{*}$ and $J_{\text {darkto }}$ as a function of absorber doping density for $T=200 \mathrm{~K}$. It is apparent that the $\mathrm{nBn}$ and $p$ - $n$ photodiode are not able to perform at $T=200 \mathrm{~K}$, whereas 
the $D^{*}$ in $\mathrm{nBnn}^{+}$and $\mathrm{pnn}^{+}$can go beyond the BLIP limit and are able to operate at high temperatures. The most important point is that the absorber layer doping density for both $\mathrm{nBnn}^{+}$and $\mathrm{pnn}^{+}$should be kept below $3 \times 10^{15} \mathrm{~cm}^{-3}$ and ideally close to $10^{14} \mathrm{~cm}^{-3}$, to minimise the tunneling current and Auger GR current. This is because increasing the temperature also increases the intrinsic carrier density; therefore, the Auger-1 lifetime becomes smaller. This is why the absorber doping density tolerable at $T=200 \mathrm{~K}$ is smaller than maximum allowed doping density at $T=100 \mathrm{~K}$.

Figures $15 \mathrm{a}$ and $\mathrm{b}$ show $D^{*}$ and $J_{\text {dark }}$ as a function of temperature for a fixed absorber doping density of $\mathrm{N}_{\mathrm{abs}}=10^{15} \mathrm{~cm}^{-3}$. It is apparent that $\mathrm{nBnn}^{+}$and $\mathrm{pnn}^{+}$photodiodes have the same level of $D^{*}$ and $J_{\text {dark }}$ at high temperatures, however $\mathrm{pnn}^{+}$performance starts to degrade at temperatures below $100 \mathrm{~K}$, because of strong BTBT. It is also apparent that dark current in $\mathrm{nBnn}^{+}$and $\mathrm{pnn}^{+}$structures at high temperatures becomes very close to the values predicted by Rule-07, which implies that HgCdTe-based HOT detectors have the potential to achieve the ultimate dark current limit predicted by Rule-07 [22, 26].
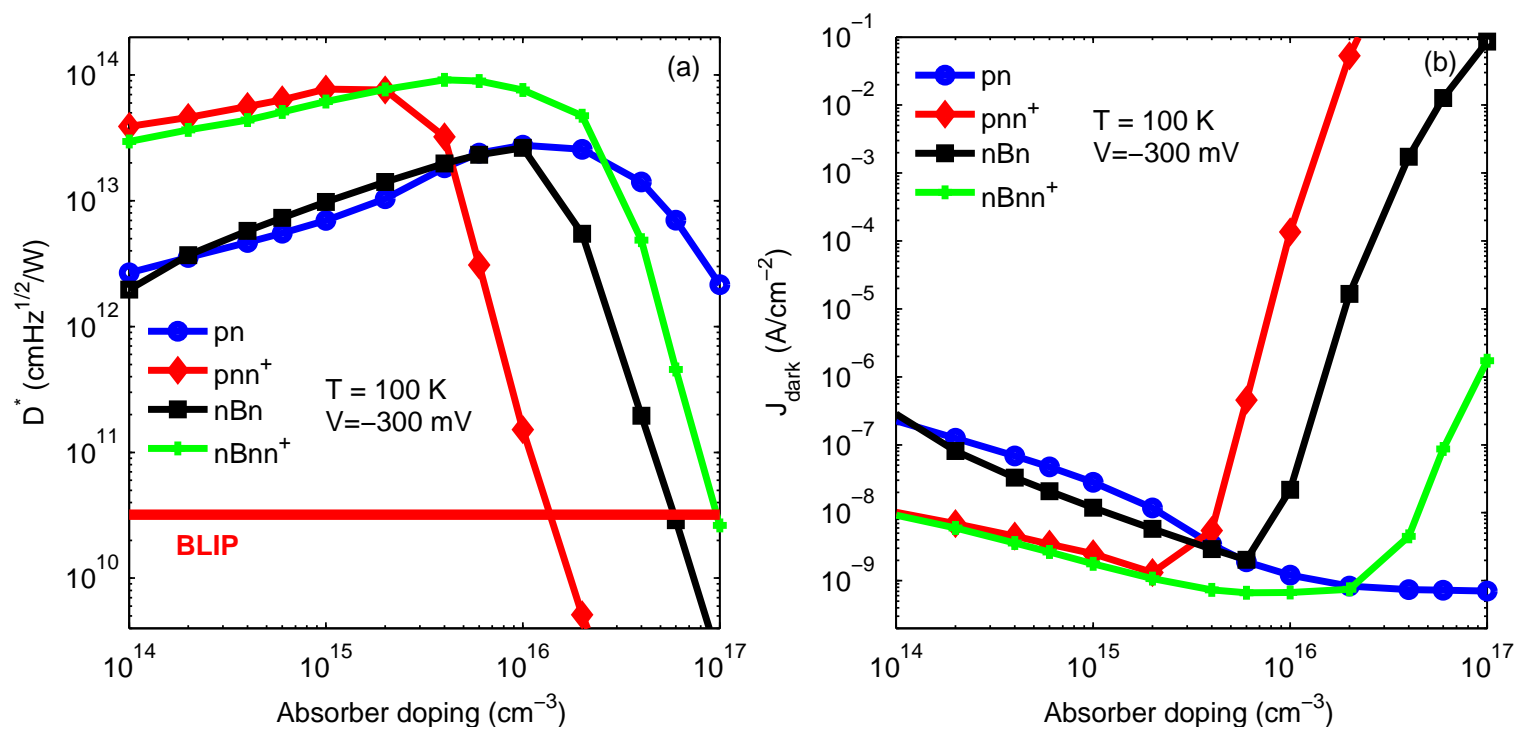

Figure 13. (a) $D^{*}$ and, (b) $J_{\text {dark }}$ as a function of absorber layer doping density for different structures at $T=100 \mathrm{~K}$ and reverse bias fixed at $-300 \mathrm{mV}$. 

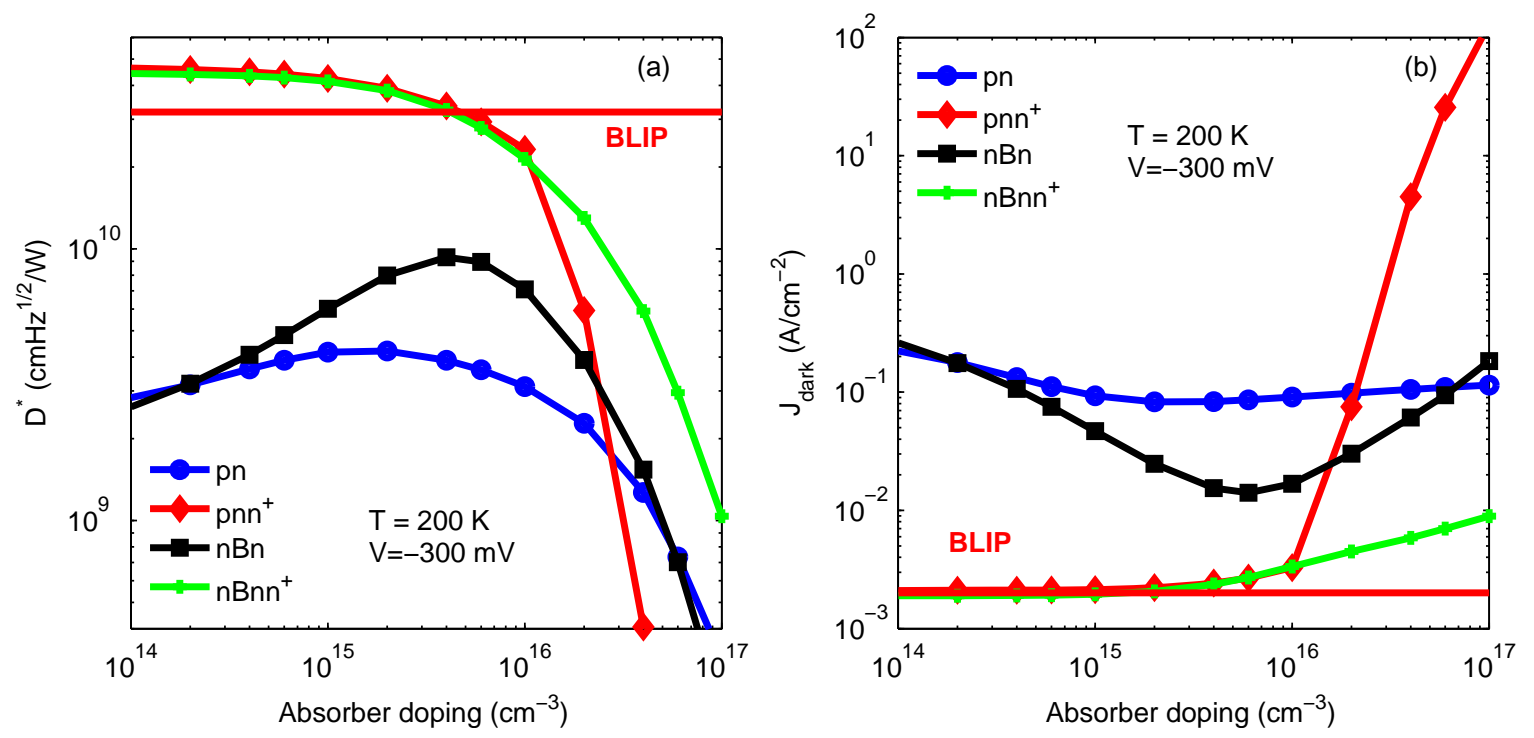

Figure 14. (a) $D^{*}$ and, (b) $J_{d a r k}$ as a function of absorber doping layer density for different structures at $T=200 \mathrm{~K}$ and reverse bias fixed at $300 \mathrm{mV}$.
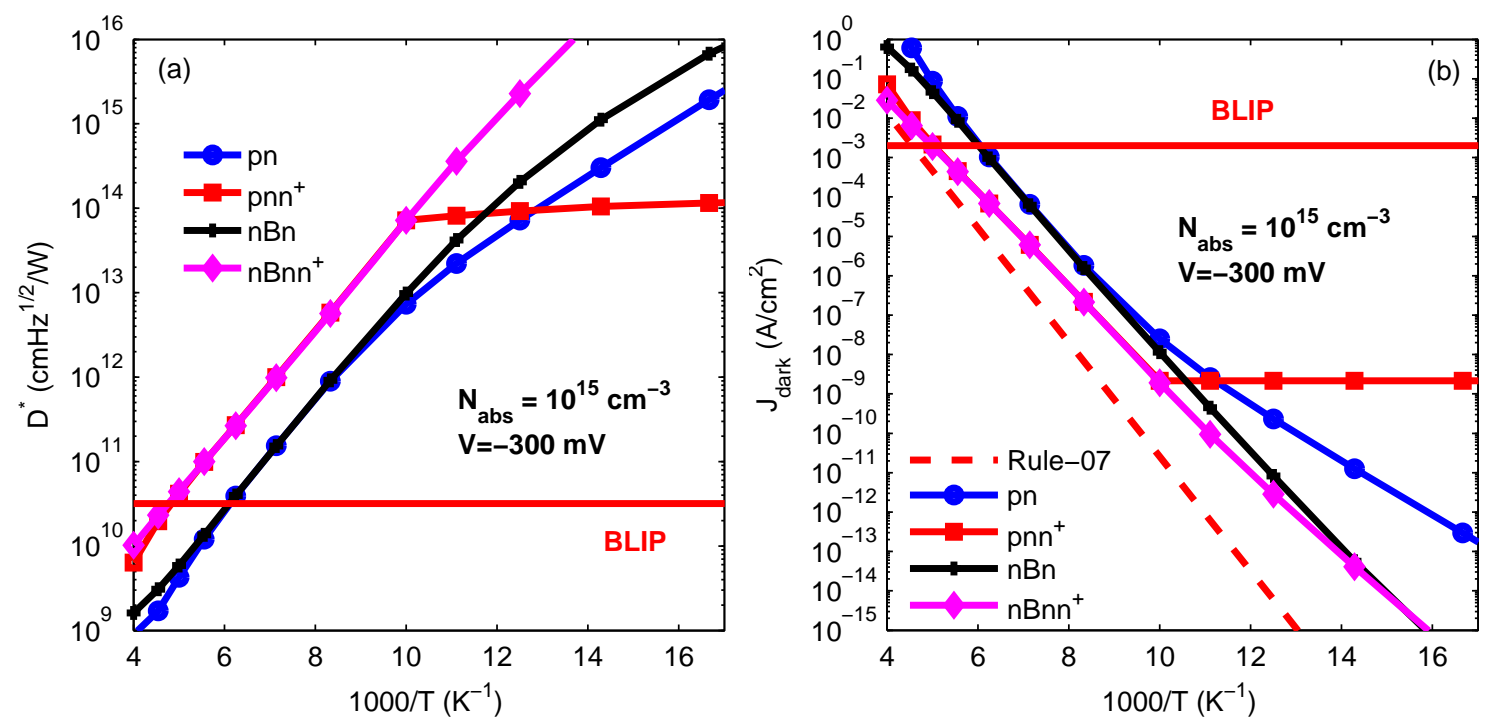

Figure 15. (a) $D^{*}$ as a function of inverse temperature with an absorber doping density of $\mathrm{N}_{\mathrm{abs}}=10^{15} \mathrm{~cm}^{-3}$, (b) $J_{\text {dark }}$ as a function of inverse temperature with an absorber doping density of Nabs $=10^{15} \mathrm{~cm}^{-3} . \mathrm{nBnn}^{+}$and $\mathrm{pnn}^{+}$can reach the dark current predicted by Rule- 07 . 


\section{CONCLUSIONS}

We performed a theoretical study of the performance of HgCdTe-based MWIR unipolar barrier detectors for elevated temperature operation, including all the important generation recombination mechanisms (SRH, Auger, radiative, BTBT, TAT, etc.). After optimizing the barrier parameters of $\mathrm{nBnn}^{+}$detector, we found that the performance of this detector is the same as that of compared to the best $\mathrm{pnn}^{+}$photodiode technology for high operatingtemperature conditions $(T>200 \mathrm{~K})$. However, the $\mathrm{nBnn}^{+}$detector is superior to $\mathrm{pnn}^{+}$ photodiode from the perspective of fabrication, because it does not involve $p$-type doping and, hence, is more uniform and requires less stringent surface passivation.

\section{ACKNOWLEDGMENTS}

The authors wish to acknowledge the Australian Research Council for financial support of this work under the Super Science Fellowship program (grant number FS110200022) and the Discovery Project program (grant number DP120104835). 


\section{APPENDIX I}

\section{Bandgap}

$E_{g}(x, T)=-0.302+1.93 x-0.81 x^{2}+0.832 x^{3}+\left(5.3510^{-4}(1-2 x) * T\right)$

\section{Electron affinity}

$\chi=4.23-0.813\left(E_{g}-0.083\right)$

\section{Intrinsic carrier concentration}

$n_{i}=(5.585-3.82 x+0.001753 T-0.001364 x T) 10^{14} E_{g}^{0.75} T^{1.5} \exp \left[\frac{-q E_{g}}{2 k_{B} T}\right]$

\section{Refractive index}

$\varepsilon_{r}=20.5-15.5 x+5.7 x^{2} ; \quad$ low frequency

$\varepsilon_{\infty}=15.2-15.5 x+5.7 x^{2} ; \quad$ high frequency

\section{Electron and hole effective masses}

$$
\begin{aligned}
& \frac{m_{0}}{m_{e}^{*}}=1+2 F+0.33 E p\left[\frac{2}{E_{g}}+\frac{1}{E_{g}+\Delta}\right] \\
& \Delta=0.893+0.165 x ; \quad F=-0.8 ; \quad E p=19 ; \quad m_{h}=0.5 m_{0}
\end{aligned}
$$

\section{SRH generation-recombination rate:}

$$
\begin{aligned}
& (G-U)_{S R H}=\frac{p n-n_{i}^{2}}{\tau_{p 0}\left[n+n_{i} \exp \left(\frac{E_{t}}{k_{B} T}\right)\right]+\tau_{n 0}\left[p+n_{i} \exp \left(\frac{-E_{t}}{k_{B} T}\right)\right]} \\
& \tau_{n 0}=\left(\sigma_{n} v_{t h, n} N_{\text {trap }}\right)^{-1} \quad \tau_{p 0}=\left(\sigma_{p} v_{t h, p} N_{\text {trap }}\right)^{-1} \\
& v_{t h, n}=\left(8 k_{B} T / \pi m_{e}^{*}\right)^{1 / 2} \quad v_{t h, p}=\left(8 k_{B} T / \pi m_{h}^{*}\right)^{1 / 2}
\end{aligned}
$$

where $E_{\text {trap }}$ is the difference between the defect level and intrinsic level. 
SRH Doping Dependence (Scharffeter relation):

$$
\tau_{d o p}\left(N_{A}, N_{D}\right)=\tau_{\min }+\frac{\tau_{\max }-\tau_{\min }}{1+\left(\frac{N_{A}+N_{D}}{N_{\text {ref }}}\right)^{\gamma}}
$$

where $\mathrm{N}_{\mathrm{A}}, \mathrm{N}_{\mathrm{D}}$ are the acceptor, donor doping concentrations. The parameters $\gamma$ and $N_{\text {ref }}$ are equal to 1 and $10^{17} \mathrm{~cm}^{-3}$.

\section{Auger generation-recombination rate:}

$$
(G-U)_{\text {Auger }}=\frac{C_{n} n+C_{p} p}{p n-n_{i}^{2}}
$$

$C_{n}=\frac{\left(\frac{m_{e}^{*}}{m_{0}}\right)\left|F_{1} F_{2}\right|^{2}}{2 n_{i}^{2} 3.8 e^{-18} \varepsilon_{\infty}^{2}(1+\mu)^{\frac{1}{2}}(1+2 \mu)} \times\left(\frac{E_{g}}{k_{B} T}\right)^{-\frac{3}{2}} \exp \left[-\left(\frac{1+2 \mu}{1+\mu}\right) \frac{E_{g}}{k_{B} T}\right]$

$$
C_{p}=C_{n}\left[\frac{1-\frac{3 E_{g}}{k_{B} T}}{1-\frac{5 E g}{4 k_{B} T}}\right]
$$

where,

$\mu=\frac{m_{e}^{*}}{m_{h}^{*}} \quad\left|F_{1} F_{2}\right|^{2}=0.2$

\section{Radiative generation-recombination rate:}

$$
\begin{aligned}
& (G-U)_{R}=G_{R}\left(p n-n_{i}^{2}\right) \\
& G_{R}=\frac{(2 \pi)^{3 / 2}}{3} \frac{h\left(\varepsilon_{\infty}\right)^{1 / 2} q^{2}}{4 \pi \varepsilon_{0} m^{2} c^{2}}\left(\frac{m_{0}}{m_{e}^{*}+m_{h}}\right)^{3 / 2} \\
& \times\left(1+\frac{m_{0}}{m_{e}^{*}}+\frac{m_{0}}{m_{h}^{*}}\right) \frac{E_{g}^{2}}{\left(k_{B} T\right)^{3 / 2}\left(m_{0} c^{2}\right)^{1 / 2}}
\end{aligned}
$$




\section{Electron-hole mobility:}

$\mu_{n}=\frac{9 \times 10^{8} b}{T^{2 a}} ; b=\left(\frac{0.2}{x}\right)^{0.75} ; a=\left(\frac{0.2}{x}\right)^{0.6}$

$\mu_{h}=0.01 \times \mu_{n}$

Mobility field dependence (extended Canali model):

$\mu_{n, p}(F)=\frac{\mu_{o-n, p}}{\left[1+\left(\frac{\mu_{o-n, p} F_{h f s}}{v_{s a t}}\right)^{\beta}\right]^{1 / \beta}}$

$v_{\text {sat }}=10^{5} \sqrt{\frac{300}{T}} \quad \beta=\left(\frac{T}{300}\right)^{\beta_{o}}$

where $\mu_{0}, v_{\mathrm{sat}}$ and $F_{\mathrm{hfs}}$ are low-filed mobility, saturation velocity and driving field, respectively. Parameter $\beta_{0}$ is a fitting coefficient in a range of 1.2 to 1.6.

Trap-assisted tunneling generation-recombination rate:

$$
\begin{aligned}
& R_{T A T}=\frac{p n-n_{i}^{2}}{\frac{\tau_{p 0}}{1+\Gamma_{p}}\left[n+n_{i} \exp \left(\frac{E_{t}}{k_{B} T}\right)\right]+\frac{\tau_{n 0}}{1+\Gamma_{n}}\left[p+n_{i} \exp \left(\frac{-E_{t}}{k_{B} T}\right)\right]} \\
& \tau_{n 0}=\left(\sigma_{n} v_{t h, n} N_{t r a p}\right)^{-1} \text { and } \tau_{p 0}=\left(\sigma_{p} v_{t h, p} N_{t r a p}\right)^{-1} \\
& v_{t h, n}=\left(8 k_{B} T / \pi m_{n}^{*}\right)^{1 / 2} \text { and } v_{t h, p}=\left(8 k_{B} T / \pi m_{p}^{*}\right)^{1 / 2}
\end{aligned}
$$

where, $\sigma_{\mathrm{n}}, \sigma_{\mathrm{p}}$ are electron, hole capture cross section, and $v^{\mathrm{n}}, v^{\mathrm{p}}$ are electron, hole thermal velocities. $\Gamma_{\mathrm{n}, \mathrm{p}}$ depends on the electron/hole effective masses.

BTBT generation-recombination rate:

$$
(G-U)_{B T B T}=\frac{q^{2} \sqrt{m^{*}} F^{2}}{4 \sqrt{2} \pi^{2} \hbar^{2} E_{g}^{1 / 2}} \exp \left(-\frac{4 \sqrt{2 m^{*}} E_{g}^{3 / 2}}{3 q \hbar F}\right)
$$

where $F$ is the Electric field. 


\section{REFERENCES}

[1] A. Rogalski, J. Antoszewski, and L. Faraone, "Third-generation infrared photodetector arrays," Journal of Applied Physics, vol. 105, p. 091101, 2009.

[2] E. C. Piquette, D. D. Edwall, D. L. Lee, and J. M. Arias, "Precise arsenic doping of $\mathrm{HgCdTe}$ by MBE and effects on compositional interdiffusion," Journal of Electronic Materials, vol. 35, pp. 1346-1349, 2006.

[3] S. Maimon and G. W. Wicks, "nBn detector, an infrared detector with reduced dark current and higher operating temperature," Applied Physics Letters, vol. 89, p. $151109,2006$.

[4] P. Martyniuk, M. Kopytko, and A. Rogalski, "Barrier infrared detectors," OptoElectronics Review, vol. 22, pp. 127-146, 2014.

[5] L. O. Bubulac, W. E. Tennant, R. A. Riedel, and T. J. Magee, "Behavior of implantation-induced defects in HgCdTe," Journal of Vacuum Science \&amp; Technology, vol. 21, pp. 251-254, 1982.

[6] A. C. Chen, M. Zandian, D. D. Edwall, R. E. De Wames, P. S. Wijewarnasuriya, J. M. Arias, et al., "MBE growth and characterization of in situ arsenic doped HgCdTe," Journal of Electronic Materials, vol. 27, pp. 595-599, 1998.

[7] M. Zandian, A. C. Chen, D. D. Edwall, J. G. Pasko, and J. M. Arias, "p-type arsenic doping of Hg1-xCdxTe by molecular beam epitaxy," Applied Physics Letters, vol. 71, pp. 2815-2817, 1997.

[8] L. O. Bubulac, "Defects, diffusion and activation in ion implanted HgCdTe," Journal of Crystal Growth, vol. 86, pp. 723-734, 1990.

[9] A. I. D'Souza, J. Bajaj, R. E. De Wames, D. D. Edwall, P. S. Wijewarnasuriya, and N. Nayar, "MWIR DLPH HgCdTe photodiode performance dependence on substrate material," Journal of Electronic Materials, vol. 27, pp. 727-732, 1998.

[10] A. M. Itsuno, J. D. Phillips, and S. Velicu, "Mid-wave infrared HgCdTe nBn photodetector," Applied Physics Letters, vol. 100, p. 161102, 2012.

[11] D. Z. Ting, C. J. Hill, A. Soibel, J. Nguyen, S. A. Keo, M. C. Lee, et al., "Antimonide-based barrier infrared detectors," 2010, pp. 76601R-76601R-14.

[12] A. Rogalski, "Recent progress in infrared detector technologies," Infrared Physics \& Technology, vol. 54, pp. 136-154, 2011.

[13] P. Klipstein, ""XBn" barrier photodetectors for high sensitivity and high operating temperature infrared sensors," in Proc. SPIE, 2008, p. 69402U.

[14] A. Rogalski and P. Martyniuk, "InAs/GaInSb superlattices as a promising material system for third generation infrared detectors," Infrared Physics \& Technology, vol. 48, pp. 39-52, 2006.

[15] B.-M. Nguyen, D. Hoffman, P.-Y. Delaunay, and M. Razeghi, "Dark current suppression in type II InAs/GaSb superlattice long wavelength infrared photodiodes with M-structure barrier," Applied Physics Letters, vol. 91, pp. -, 2007.

[16] M. Kopytko, J. Wróbel, K. Jóźwikowski, A. Rogalski, J. Antoszewski, N. D. Akhavan, et al., "Engineering the Bandgap of Unipolar HgCdTe-Based nBn Infrared Photodetectors," Journal of Electronic Materials, vol. 44, pp. 158-166, 2015/01/01 2015.

[17] N. D. Akhavan, G. Jolley, G. A. Umana-Membreno, J. Antoszewski, and L. Faraone, "Design of Band Engineered $\mathrm{HgCdTe} n \mathrm{nBn}$ Detectors for MWIR and LWIR Applications," Electron Devices, IEEE Transactions on, vol. 62, pp. 1-1, 2015.

[18] N. D. Akhavan, G. A. Umana-Membreno, G. Jolley, J. Antoszewski, and L. Faraone, "A method of removing the valence band discontinuity in $\mathrm{HgCdTe}$-based $\mathrm{nBn}$ detectors," Applied Physics Letters, vol. 105, pp. -, 2014. 
[19] E. F. Schubert, L. W. Tu, G. J. Zydzik, R. F. Kopf, A. Benvenuti, and M. R. Pinto, "Elimination of heterojunction band discontinuities by modulation doping," Applied Physics Letters, vol. 60, pp. 466-468, 1992.

[20] M. Kopytko, "Design and modelling of high-operating temperature MWIR HgCdTe nBn detector with n- and p-type barriers," Infrared Physics \& Technology, vol. 64, pp. 47-55, 5// 2014.

[21] G. Masetti, M. Severi, and S. Solmi, "Modeling of carrier mobility against carrier concentration in arsenic-, phosphorus-, and boron-doped silicon," Electron Devices, IEEE Transactions on, vol. 30, pp. 764-769, 1983.

[22] C. Canali, G. Gavioli, A. Losi, and G. Ottaviani, "HOT-HOLE DIFFUSIVITY IN GE AT 77K," Solid State Communications, vol. 20, pp. 57-60, 19761976.

[23] H. F. Schaake, M. A. Kinch, D. Chandra, F. Aqariden, P. K. Liao, D. F. Weirauch, et al., "High-Operating-Temperature MWIR Detector Diodes," Journal of Electronic Materials, vol. 37, pp. 1401-1405, 2008.

[24] P. Martyniuk, W. Gawron, and A. Rogalski, "Theoretical Modeling of HOT HgCdTe Barrier Detectors for the Mid-Wave Infrared Range," Journal of Electronic Materials, vol. 42, pp. 3309-3319, 2013.

[25] W. E. Tennant, D. Lee, M. Zandian, E. Piquette, and M. Carmody, "MBE HgCdTe Technology: A Very General Solution to IR Detection, Described by "Rule 07", a Very Convenient Heuristic," Journal of Electronic Materials, vol. 37, pp. 1406-1410, 2008.

[26] T. J. De Lyon, J. E. Jensen, I. Kasai, G. M. Venzor, K. Kosai, J. B. de Bruin, et al., "Molecular-beam epitaxial growth and high-temperature performance of $\mathrm{HgCdTe}$ midwave infrared detectors," Journal of Electronic Materials, vol. 31, pp. 220-226, 2002.

[27] W. Scott, "Electron Mobility in Hg1-xCdxTe," Journal of Applied Physics, vol. 43, pp. 1055-1062, 1972.

[28] M. Kopytko, A. Kębłowski, W. Gawron, P. Madejczyk, A. Kowalewski, and K. Jóźwikowski, "High-operating temperature MWIR nBn HgCdTe detector grown by MOCVD," Opto-Electronics Review, vol. 21, pp. 402-405, 2013.

[29] J. Rothman, J. Meilhan, G. Perrais, J.-P. Belle, and O. Gravrand, "Maximum entropy mobility spectrum analysis of $\mathrm{HgCdTe}$ heterostructures," Journal of Electronic Materials, vol. 35, pp. 1174-1184, 2006.

[30] S. Velicu, J. Zhao, M. Morley, A. M. Itsuno, and J. D. Phillips, "Theoretical and experimental investigation of MWIR HgCdTe nBn detectors," 2012, pp. 82682X82682X-13.

[31] P. Martyniuk and A. Rogalski, "Modelling of MWIR HgCdTe complementary barrier HOT detector," Solid-State Electronics, vol. 80, p. 96, 2012.

[32] A. M. Itsuno, J. D. Phillips, and S. Velicu, "Design and Modeling of HgCdTe nBn Detectors," Journal of Electronic Materials, vol. 40, pp. 1624-1629, 2011.

[33] A. Itsuno, J. Phillips, and S. Velicu, "Design of an Auger-Suppressed Unipolar HgCdTe NBvN Photodetector," Journal of Electronic Materials, vol. 41, pp. 28862892, 2012.

[34] S. J. Elliott and P. A. Nelson, Algorithm for multichannel LMS adaptive filtering vol. 21: Institution of Engineering and Technology, 1985. 